NOTAS CIENTÍFICAS

\title{
LEVANTAMENTO DA ENTOMOFAUNA ASSOCIADA A CULTIVARES DE BATATA EM DUAS ÉPOCAS DE CULTIVO ${ }^{1}$
}

\author{
ANDERSONDIONEI GRÜTZMACHER ${ }^{2}$ e DIONISIO LINK
}

\begin{abstract}
RESUMO - Foi feito um levantamento da população de artrópodes num agroecossistema de batata, Solanum tuberosum L., em Santa Maria, RS, durante o ano agrícola 1989/90. O objetivo desta pesquisa foi comparar a entomofauna em diferentes cultivares de batata durante a época tradicional do seu crescimento e na safrinha. $\mathrm{O}$ experimento consistiu de nove cultivares durante a época tradicional, e de dez cultivares durante a safrinha. Amostras de insetos foram coletadas semanalmente com a rede de varredura (15 redadas por amostra). Os coleópteros foram predominantes durante a época tradicional do crescimento da cultura, e os dípteros, na safrinha. As espécies fitófagas mais freqüentes foram: Epitrix sp., na época tradicional, e Diabrotica speciosa (Germar), na safrinha, seguidas da Empoasca kraemeri Ross \& Moore, em ambos os períodos de crescimento dessa cultura. Foi coletado um número maior de insetos no período tradicional de crescimento da cultura do que na safrinha. Os insetos mostraram preferência pela cultivar Bintje durante o período tradicional, e pela Pomina, na safrinha. A cultivar Sahael foi a menos procurada, nos dois períodos. As espécies de predadores Eriopis connexa (Germar) e Geocoris sp. foram as mais numerosas no período tradicional de crescimento da cultura. Durante a safrinha, a Cycloneda sanguinea (L.) predominou em todas as cultivares.
\end{abstract}

\section{SURVEY OF THE ENTOMOFAUNA ASSOCIATED WITH POTATO VARIETIES DURING TWO CROP SEASONS}

ABSTRACT - A survey of arthropods present in a potato (Solanum tuberosum L.) agroecossystem was conducted in Santa Maria, RS, Brazil, during 1989/90 field season. The objective of this research was to compare the entomofauna in different potato cultivars during the traditional and off-season time for potato growing. The experiment consisted of nine cultivars during the traditional season and ten cultivars during the off-season time. Insect samples were taken weekly with a sweep net ( 15 nets per sample). Coleoptera was the predominant insect order during the traditional potato growing season, and Diptera during the off-season. Epitrix sp. was quantitatively the most important in the traditional season, and Diabrotica speciosa (Germar) in the off-sea-

\footnotetext{
${ }^{1}$ Aceito para publicação em 19 de abril de 1999.

${ }^{2}$ Eng. Agrôn., M.Sc., Prof. Assistente, Dep. de Fitossanidade, UFPel, Caixa Postal 354, CEP 96010-900 Pelotas, RS.E-mail: adgrutz@ufpel.tche.br

${ }^{3}$ Eng. Agrôn., Ph.D., Prof. Titular, Centro de Ciências Rurais, UFSM, CEP 97105-900 Santa Maria, RS. E-mail: dlink@ ccr.ufsm.br
} 


\begin{abstract}
son, followed by Empoasca kraemeri Ross \& Moore in both potato growing season. Greater number of insects was collected during the traditional season than off-season for growing potatoes. Insects preferred Bintje cultivar during the traditional season and Pomina cultivar during the off-season time. Sahael cultivar was the least preferred in both potato growing season. Eriopis connexa (Germar) and Geocoris sp. were the most abundant predator species in the traditional potato growing season. During the off-season, Cycloneda sanguinea (L.) was the predominant species in all cultivars.
\end{abstract}

Apesar de a batata ser cultivada desde longa data, pouco se sabe sobre a entomofauna da cultura e a preferência dos insetos em relação às cultivares. A utilização de produtos químicos para o controle dos insetos que danificam a cultura tem sido preconizada como uma das únicas medidas de controle, pela maioria dos autores. Entretanto, a necessidade do conhecimento qualiquantitativo da entomofauna da batata é fundamental para a avaliação de danos e estabelecimento de um programa de manejo de pragas. É importante identificar os insetos que realmente são pragas, aqueles que, apesar de fitófagos, não chegam a causar danos, e, finalmente, os insetos úteis (parasitóides, predadores e polinizadores) que convivem no mesmo agroecossistema.

Orlando (1965) referiu como prejudiciais à cultura da batata 15 espécies de insetos, sendo sete da ordem Coleoptera, três da ordem Lepidoptera e da Homoptera, e um da ordem Hymenoptera e da Diptera. Foram relacionadas por Biezanko et al. (1974) 20 espécies de lagartas alimentando-se da planta da batata.

Barbosa \& França (1981) destacaram como pragas mais nocivas à parte aérea o pulgão-verde (Myzus persicae (Sulzer), a vaquinha (Diabrotica sp.), o burrinho-da-batatinha (Epicauta spp.), a traça (Phthorimaea operculella (Zeller) e o minador-de-folhas (Liriomyza spp.).

Radcliffe (1982) realizou uma revisão sobre as principais pragas da cultura da batata no mundo. Foram reportados como importantes transmissores de viroses os pulgões, principalmente a espécie Myzus persicae (Sulzer). As cigarrinhas da família Cicadellidae foram citadas como pragas muito comuns nos trópicos e subtrópicos, principalmente a Empoasca fabae (Harris). Citou ainda vários insetos desfolhadores, entre os mais importantes Epitrix spp., Epilachna sp., Diabrotica spp., Epicauta spp., Phthorimaea operculella (Zeller), Liriomyza spp. e Leptinotarsa decemlineata (Say), que é considerada a praga mais perniciosa nos Estados Unidos.

Juruena \& Meyer-Cachapuz (1987) catalogaram como nocivas à cultura da batata, no Rio Grande do Sul, nove espécies de insetos.

Como espécies daninhas à cultura da batata foram referidas por Gallo et al. (1988) quatro espécies de lagartas (Lepidoptera), seis espécies de Coleoptera, uma larva de Diptera e quatro espécies de Homoptera. Hohmann (1989) referiu que as espécies-pragas encontradas em maior número foram os afídeos (entre eles Myzus persicae (Sulzer) e Macrosiphum euphorbiae (Thomas)), além de Epitrix spp., Diabrotica speciosa (Germar) e Empoasca kraemeri Ross \& Moore. 
Este trabalho objetivou avaliar o comportamento da entomofauna em coleção de cultivares de batata, na safra, época tradicional de cultivo, ou seja, plantio em agosto-setembro, e na safrinha, plantio fora de época, ou seja, fevereiro-março, observando a possível preferência varietal e os aspectos quali-quantitativos dos inimigos naturais.

O experimento foi realizado no ano agrícola 1989/90 através de levantamentos de artrópodes presentes na cultura da batata (Solanum tuberosum), no munícipio de Santa Maria, RS. Os levantamentos foram realizados na cultura da safra, nas seguintes cultivares: Bronca, Mariana, Apollo, Frisia, Bintje, Radosa, Korrigane, Sahael e Baronesa; e da safrinha: Mistral, Pomina, Apollo, Korrigane, Yesmina, Mariana, Sahael, Rústica, Planta e Baronesa.

A amostragem nas culturas da safra teve início em 10/11/89 e término em $15 / 12 / 89$, e os de safrinha tiveram início em 20/4/90 e término em $7 / 6 / 90$. As amostragens foram realizadas semanalmente, usando-se rede de varredura, segundo o modelo descrito por Beall (1935). Efetuaram-se 15 redadas por amostra, perfazendo um total de 216 amostras no período de safra, e de 320 amostras no período de safrinha.

Cada cultivar teve quatro repetições, e o delineamento experimental foi de blocos ao acaso.

Os artrópodes coletados foram levados ao Laboratório de Entomologia do Departamento de Defesa Fitossanitária, Centro de Ciências Rurais, Universidade Federal de Santa Maria, onde foi feita triagem, quantificação e identificação, inicialmente por ordem e família, posteriormente por espécie, realizada por especialistas de cada grupo.

Os artrópodes coletados na safra e posteriormente identificados foram agrupados em 10 ordens, com um total de 10.792 exemplares (Tabela 1).

Os artrópodes coletados na cultura da safrinha resultaram em 11 ordens, com um total de 5.262 exemplares (Tabela 2).

TABELA 1. Número e porcentagem de indivíduos, de diferentes ordens de artrópodes, coletados em plantas de nove cultivares de Solanum tuberosum (batata) no período de safra. Santa Maria, RS, 1989.

\begin{tabular}{|c|c|c|c|c|c|c|c|c|c|c|c|}
\hline \multirow[t]{2}{*}{ Ordem } & \multicolumn{9}{|c|}{ Cultivar $^{1}$} & \multirow[t]{2}{*}{ Total } & \multirow{2}{*}{$\begin{array}{c}\text { Porcen- } \\
\text { tagem }\end{array}$} \\
\hline & 1 & 2 & 3 & 4 & 5 & 6 & 7 & 8 & 9 & & \\
\hline Araneae & 5 & 1 & 1 & 1 & 2 & 1 & 1 & 3 & 2 & 17 & 0,16 \\
\hline Coleoptera & 580 & 381 & 295 & 647 & 1.030 & 566 & 589 & 262 & 596 & 4.946 & 45,83 \\
\hline Dermaptera & 1 & - & - & - & - & - & 1 & - & - & 2 & 0,02 \\
\hline Diptera & 129 & 52 & 87 & 132 & 111 & 93 & 108 & 69 & 173 & 954 & 8,84 \\
\hline Hemiptera-Het. & 46 & 24 & 35 & 61 & 80 & 42 & 53 & 29 & 67 & 437 & 4,05 \\
\hline Hemiptera-Hom. & 579 & 296 & 285 & 506 & 722 & 512 & 504 & 325 & 650 & 4.379 & 40,58 \\
\hline Hymenoptera & - & 1 & - & 4 & 3 & 1 & - & 1 & 9 & 19 & 0,17 \\
\hline Lepidoptera & 1 & - & - & - & - & 1 & - & 1 & 4 & 7 & 0,06 \\
\hline Neuroptera & 1 & - & 1 & - & 1 & - & - & - & - & 3 & 0,03 \\
\hline Orthoptera & 3 & 3 & 3 & 5 & 1 & 3 & 3 & 3 & 4 & 28 & 0,26 \\
\hline Total & 1.345 & 758 & 707 & 1.356 & 1.949 & 1.220 & 1.259 & 693 & 1.505 & 10.792 & 100 \\
\hline Porcentagem & 12,46 & 7,02 & 6,55 & 12,56 & 18,06 & 11,30 & 11,67 & 6,42 & 13,94 & 100 & \\
\hline
\end{tabular}

\footnotetext{
${ }^{1}$ Cultivares: 1: Bronca; 2: Mariana; 3: Apollo; 4: Frisia; 5: Bintje; 6: Radosa; 7: Korrigane; 8: Sahael;
} 9: Baronesa. 
Tanto no período da safra, como de safrinha se destacaram quatro ordens de insetos, sendo que no período da safra as ordens Coleoptera e HemipteraHomoptera foram, respectivamente, as mais numerosas, com 45,83\% e 40,58\% do total de artrópodes, e seguidas pelas ordens Diptera e HemipteraHeteroptera com $8,84 \%$ e $4,05 \%$, respectivamente (Tabela 1). As demais ordens ocorreram em porcentuais bastante baixos, com valores inferiores a $1 \%$. No período de safrinha, as ordens Diptera e Hemiptera-Homoptera foram as mais numerosas, com $43,92 \%$ e $30,08 \%$ do total de artrópodes, respectivamente (Tabela 2). Seguiu-se a ordem Coleoptera, com 21,28\% e HemipteraHeteroptera com $3,44 \%$. As outras ordens foram coletadas com valores extremamente baixos, com porcentuais abaixo de $1 \%$, semelhante ao que ocorreu na safra. Durante a época de safra, na ordem Coleoptera, se destacou a família Chrysomelidae, com 94,90\% dos besouros coletados; Epitrix sp. foi o mais abundante.

No período de safrinha também a família Chrysomelidae, com 92,59\% dos coleópteros, foi a mais importante; Diabrotica speciosa foi a mais abundante. Tanto no período de safra, como no de safrinha, se destacou quantitativamente na ordem Hemiptera-Homoptera, da família Cicadellidae, a espécie Empoasca kraemeri. Resultados similares foram obtidos por Hohmann (1989) durante as safras da seca e das águas, utilizando-se bandejas de alumínio com água; foram encontradas também como espécies predominantes a pulga-do-fumo, Epitrix spp., e a vaquinha Diabrotica speciosa. Já a cigarrinha-verde, Empoasca kraemeri, somente ocorreu na safra da seca. Foi observado, por esse autor, maior população de insetos durante o plantio da batata na safra da seca.

Nas cultivares de safra teve-se a formação de três níveis diferenciados, tendo sido Bintje a cultivar preferida, com 18,06\% do total de artrópodes

TABELA 2. Número e porcentagem de indivíduos, de diferentes ordens de artrópodes, coletados em plantas de 10 cultivares de Solanum tuberosum (batata) no período de safrinha. Santa Maria, RS, 1990.

\begin{tabular}{|c|c|c|c|c|c|c|c|c|c|c|c|c|}
\hline \multirow[t]{2}{*}{ Ordem } & \multicolumn{10}{|c|}{ Cultivar $^{1}$} & \multirow[t]{2}{*}{ Total } & \multirow{2}{*}{$\begin{array}{c}\text { Porcen- } \\
\text { tagem }\end{array}$} \\
\hline & 1 & 2 & 3 & 4 & 5 & 6 & 7 & 8 & 9 & 10 & & \\
\hline Araneae & 2 & 4 & & 1 & - & - & - & 2 & 2 & - & 11 & 0,21 \\
\hline Coleoptera & 127 & 134 & 99 & 129 & 153 & 106 & 58 & 95 & 91 & 128 & 1.120 & 21,28 \\
\hline Dermaptera & - & - & - & 1 & 1 & 1 & - & 1 & - & 1 & 5 & 0,09 \\
\hline Diptera & 119 & 261 & 182 & 234 & 192 & 272 & 187 & 234 & 346 & 284 & 2.311 & 43,92 \\
\hline Hemiptera-Het. & 12 & 21 & 7 & 17 & 20 & 16 & 9 & 32 & 23 & 24 & 181 & 3,44 \\
\hline Hemiptera-Hom. & 168 & 204 & 178 & 133 & 138 & 143 & 152 & 160 & 155 & 152 & 1.583 & 30,08 \\
\hline Hymenoptera & - & 1 & 1 & - & 1 & 4 & 1 & 1 & - & - & 9 & 0,17 \\
\hline Lepidoptera & 1 & - & - & 2 & - & - & 1 & 1 & 1 & - & 6 & 0,11 \\
\hline Neuroptera & - & - & 7 & 10 & - & - & - & 2 & - & 1 & 20 & 0,38 \\
\hline Odonata & 1 & - & - & - & - & - & - & - & - & - & 1 & 0,02 \\
\hline Orthoptera & - & 7 & - & 1 & 3 & 2 & - & 2 & - & - & 15 & 0,28 \\
\hline Total & 430 & 632 & 474 & 528 & 508 & 544 & 408 & 530 & 618 & 590 & 5.262 & 100 \\
\hline Porcentagem & 8,17 & 12,01 & 9,01 & 10,03 & 9,65 & 10,34 & 7,75 & 10,07 & 11,74 & 11,21 & 100 & \\
\hline
\end{tabular}

1 Cultivares: 1: Mistral; 2: Pomina; 3: Apollo; 4: Korrigane; 5: Yesmina; 6: Mariana; 7: Sahael; 8: Rústica; 9: Planta; 10: Baronesa. 
coletados. A cultivar Sahael, com 6,42\%, foi a menos visitada, seguida pela Apollo com 6,55\%, e Mariana com 7,02\%. Em um extrato intermediário estavam as outras cultivares, variando seus porcentuais de $11,30 \%$ até $13,94 \%$ (Tabela 1).

Nas cultivares de safrinha, houve a formação aparente de somente dois níveis preferenciais, pois devido às condições meteorológicas de inverno ocorreu a coleta de um menor número de exemplares. A cultivar preferida foi a Pomina, com $12,01 \%$ do total de artrópodes, e no nível inferior ficou a cultivar Sahael, com 7,75\%, seguida pela Mistral, com 8,17\%.

Do total de cultivares da safra e safrinha, a cultivar Baronesa foi a mais susceptível aos insetos (Fig. 1); ressalta-se que esta cultivar é a mais plantada pelos agricultores. A cultivar mais resistente foi a Sahael. Na safra coletou-se maior número de exemplares no final do ciclo da cultura, tendo o período de máxima coleta ocorrido na metade do mês de dezembro, principalmente no tocante à cultivar Bintje, que apresentou alta população de Epitrix sp. e E. kraemeri.

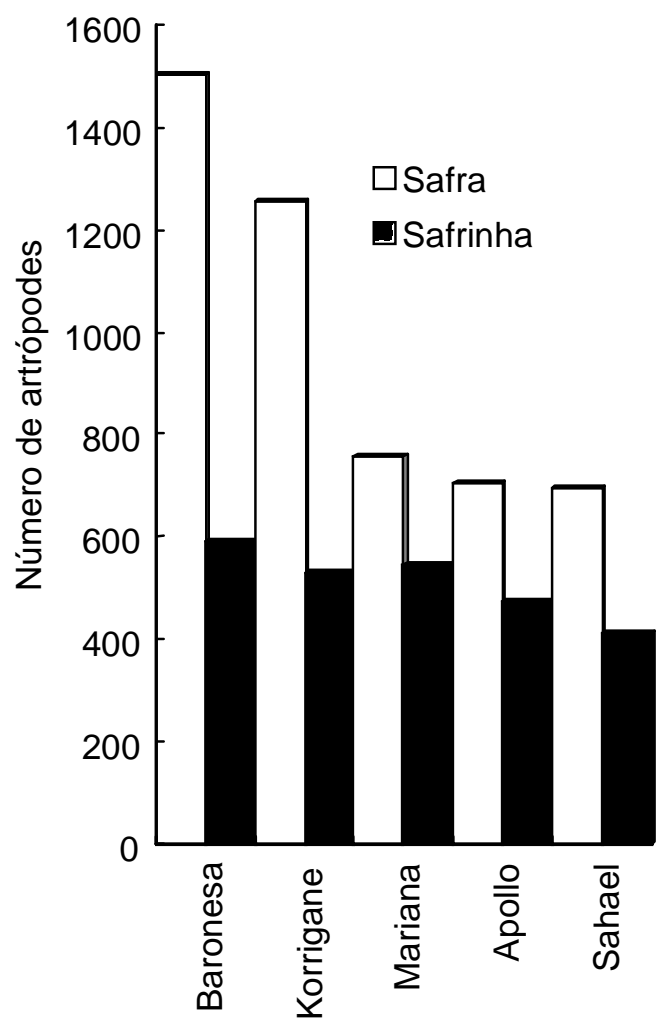

\section{Cultivar}

FIG. 1. Número total de artrópodes em relação às cultivares de batata (Solanum tuberosum) plantadas na safra e na safrinha. Santa Maria, RS, 1989/1990. 
Durante a safrinha, em que as coletas se realizaram no inverno, obteve-se grande variação no número de exemplares coletados nas diferentes datas, devido, principalmente, aos elevados valores de precipitação pluvial, $150 \mathrm{~mm}$ em 24 horas, e de variações drásticas de temperaturas, entre $2^{\circ} \mathrm{C}$ e $25^{\circ} \mathrm{C}$, em 24 horas. A ordem Diptera apresentou maior abundância de exemplares no final do ciclo. As ordens Coleoptera e Hemiptera-Homoptera, praticamente em todas as cultivares sofreram decréscimo no número de exemplares capturados nas últimas coletas.

$\mathrm{Na}$ safra, constatou-se a presença dos besouros predadores: Callida sp. e Lebia concinna (Carabidae), Cycloneda sanguinea, Eriopis connexa e Hypodamia convergens (Coccinellidae). Verificou-se a presença dos predadores Geocoris sp. e Nabis sp.(Hemiptera-Heteroptera) e Chrysopa sp. (Neuroptera).

Na safrinha, houve a ocorrência de Podisus sp., de Geocoris sp. e de Nabis sp. (Hemiptera-Heteroptera). Coletaram-se Coleomegilla quadrifasciata, Cycloneda sanguinea, Eriopis connexa, Hyperaspis sp. e Hypodamia convergens (Coccinellidae).

Na safra, E. connexa e Geocoris sp. entre todas essas espécies de predadores destacaram-se como as mais numerosas, coletadas sobre todas as cultivares. Durante a safrinha, o número de predadores coletados foi menor, destacando-se a espécie $C$. sanguinea, presente em todas as cultivares e coletada em maior número. Hohmann (1989) identificou como principais espécies de inimigos naturais na batata, C. sanguinea, Scymnus spp., Orius spp., L. concinna, E. connexa e Hyperaspis festiva, e observou-se a maior ocorrência de inimigos naturais durante a safra da seca. Do mesmo modo, Barbosa \& França (1981) relataram que C. sanguinea, E. connexa, Chrysopa sp., Geocoris sp., Nabis sp. e uma série de microhimenópteros parasitas foram coletados freqüentemente nos batatais brasileiros. Na ordem Coleoptera, na safra obteve-se a presença de Epitrix sp. como espécie muito abundante, com uma frequiência superior a $75 \%$ na média de todas as cultivares. Na safrinha, Diabrotica speciosa apresentou uma frequiência média de $70 \%$ em todas as cultivares, sendo uma espécie muito abundante na maioria delas. Segundo Hohmann (1989), essas duas espécies desfolhadoras causaram considerável prejuízo na fase inicial de desenvolvimento das plantas, mas apesar de a infestação ter sido alta, verificou que as plantas apresentaram boa capacidade de tolerância aos danos. Situação similar foi constatada neste experimento. As cultivares menos infestadas não são recomendadas, principalmente pela baixa cotação comercial no mercado.

\section{REFERÊNCIAS}

BARBOSA, S.; FRANÇA, F.H. Pragas da batata e seu controle. Informe Agropecuário, Belo Horizonte, v.7, n.76, p.55-61, abr. 1981.

BEALL, G. A study of arthropods by the method of sweeping. Ecology, San Diego, v.16, n.2, p.216-225, 1935 
BIEZANKO, C.M.; RUFFINELLI, A.; LINK, D. Plantas y otras sustancias alimenticias de las orugas de los Lepidopteros Uruguayos. Revista do Centro de Ciências Rurais, Santa Maria, v.4, n.2, p.107-148, 1974.

GALLO, D.; NAKANO, O.; SILVEIRA NETO, S.; CARVALHO, R.P.L.; BATISTA, G.C.; BERTI FILHO, E.; PARRA, J.R.P.; ZUCCHI, R.A.; ALVES, S.B.; VENDRAMIM, J.D. Manual de entomologia agrícola. 2.ed. São Paulo : Ceres, 1988. 646p.

HOHMANN, C.L. Levantamento dos Artrópodos associados à cultura da batata no município de Irati, Paraná. Anais da Sociedade Entomológica do Brasil, Porto Alegre, v.18, p.53-60, 1989. Supl.

JURUENA, L.F.; MEYER-CACHAPUZ, L.M. Pragas da batata. IPAGRO Informa, Porto Alegre, n.29, p.7-93, maio 1987.

ORLANDO, A. Principais pragas da batatinha e indicações de combate. Tudo sobre batata. Boletim de Campo, Rio de Janeiro, v.20, n.190, p.33-51, 1965.

RADCLIFFE, E.B. Insect pests of potato. Annual Review of Entomology, Palo Alto, v.27, p.173-204, 1982. 